\title{
ACADEMIC TRACK OF FOSS4G 2018 LEAVING NO-ONE BEHIND
}

\author{
A. H. Namangaya ${ }^{\mathrm{a}}$ and M. A. Brovelli ${ }^{\mathrm{b} *}$ \\ a School of Urban and Regional Planning, Ardhi University, Dar es Salaam, Tanzania, namangaya@yahoo.com \\ b Department of Civil and Environmental Engineering, Politecnico di Milano, Milano, Italy, maria.brovelli@polimi.it
}

\section{Commission IV, WG IV/4}

KEY WORDS: FOSS4G, Dar Es Salaam, Academic Track, Preface

\section{PREFACE}

The Free and Open Source Software for Geospatial Conference - FOSS4G - is an annual conference hosted by OSGeo (Open Source Geospatial Foundation: https://www.osgeo.org/) focused on building awareness, cooperation, and development of open source geospatial software. Starting in 2006, FOSS4G conferences have been held globally with the last event in Boston attracting over 1100 attendees.

2018 is a very special year for FOSS4G because, after 10 years, the event is back again to Africa. This year the conference is incorporating other global communities such as the annual summit of the HOT (Humanitarian OpenStreetMap Team: https://www.hotosm.org/) and Understanding Risk Africa (https://understandrisk.org/). By bringing together these communities a great synergy that has attracted the best and brightest developers, users and educators in the field of geospatial open science, open source, open data and open education will occur!.

As befits a diverse conference like FOSS4G, we have a strong Academic Track. It has been organized thanks to ISPRS (International Society of Photogrammetry and Remote Sensing: http://www.isprs.org ) and OSGeo. In FOSS4G, the Academic Track is about cogeneration and convergence of knowledge and theoretical development by researchers, innovations and breakthroughs by inventors, and developers who are providing solutions to the needs of users, practitioners, and companies.

The Academic Track is to helping to develop the frontier of research and innovation in the realm of open geospatial research. Because life is interdisciplinary, the Academic Track is multidisciplinary and global in composition with the commonality being in the use of open data and open source software.

Holding a FOSS4G in Dar es Salaam will leave a geospatial legacy for Tanzania and the wider African community. This will serve to aid the global OSGeo community in the years to come as well as developing the African geospatial community. Open source software, sharing platforms and open data are most influential factors is bridging the digital divide.

The opportunity of open data, open source software, and new innovative algorithms offer an unprecedented opportunity for us all to work together to not leave anyone behind. In developing their own solutions to contextual problems as well as adapt the existing algorithms, methodologies, and source code to the needs of emerging economies.

Open source software, algorithms and data are shifting the responsibility of closing the digital divide from government and large formal institutions to individuals and companies. FOSS4G is a unique occasion for bringing the awareness to African researchers and potential innovators of the possibilities; explain to the western colleagues the issues and directions where their researches and inventions are needed; and facilitate face to face meetings among individuals.

Dar es Salaam, the host city of FOSS4G, is one of the fastest growing cities in the world with the consequent problems of informal growth, social, and humanitarian challenges. The fast rate of economic growth can give a positive vibe - not only to the city but to the country and continent at large.

Therefore, while we acknowledge that FOSS4G is one of the shining lights in the constellation of stars that we find ourselves looking at, much has to be done to first attract the youth and let them know of the possibilities. The President of the World Bank mentioning, when visiting Dar es Salaam in 2017, that "it is fundamental to attract mentors from the Global North and South, to create start-ups based on open data and shared algorithms in the African business environment, and to compel large local companies to digitize their business processes and to use this digital possibilities to expand their global reach".

The composition of sponsors, papers and participants does not show very much progress on this front, except a few from South Africa. Africa as a whole is poorly represented - we need to change this and ensure that we are leaving no-one behind. It is not only in economic terms where FOSS4G can have an impact, many papers presented have humanitarian orientation, and Africa, because of its Infrastructure challenges, can use technologies based on open source inventions and software to optimize process such as building resilience communities and serve lives.

Already good results have demonstrated how Africa can innovate, such as mobile money, progress in citizen mapping platforms for resilience, and numerous other innovations. A FOSS4G in Dar es Salaam will also motivate our governments to open their policies towards open data and increase internet penetration through their policies. We believe they are represented here. Therefore, FOSS4G can be an important shining light at the door that requires to attract more African young people in future events and forge partnership while we are here.

\footnotetext{
Corresponding author
} 
On extra curricular aspects, conducting such event in Dar es Salaam will expose more to beautiful and peaceful country of Tanzania and its attractions such as Serengeti, Zanzibar beaches, Dar es Salaam and its environment and cultural diversity. These are beautiful scenery to explore and get inspired!

The theme of this FOSS4G is "Leave no-one behind". This is more than a wish. It is a commitment that the Local Organiser Committee and the Scientific Committee of the Conference have embraced to build a better future starting from today. Dar Es Salaam will be a small but important milestone in this path.

\section{FOSS4G 2018 Academic Track Scientific Committee}

Hussein Abdulmuttalib, DubaiMunicipality GIS Department, Dubai,UAE,husseinma@dm.gov.ae Andy Anderson, Amherst College, Amherst, USA, aanderson@amherst.edu Paola Carrara, IREA CNR, , Milan, Italy, carrara.p@irea.cnr.it

Marco Ciolli, Dipartimento di Ingegneria Civile Ambientale e Meccanica, Università di Trento, Trento, Italy, marco.ciolli@unitn.it Serena Coetzee, University of Pretoria, Pretoria, South Africa, serena.coetzee@up.ac.za Margherita Di Leo, European Commission, JRC, Ispra, Italy, margherita.di-leo@ec.europa.eu Nimalika Fernando, Curtin University, Perth, Australia, nimalikaf@gmail.com Aniruddha Ghosh, University of California, Davis, USA, anighosh@ucdavis.edu Lena Halounova, CTU Prague, Prague, Czech Republic, isprs-sg@isprs.org Christian Heipke, Leibniz Universitaet Hannover, Hannover, Germany, heipke@ipi.uni-hannover.de Ivana Ivanova, Curtin University, Perth, Australia, ivana.ivanova@curtin.edu.au Robbert Kiunsi, Ardhi University, Dar es Salaam, Tanzania, robertkiunsi@yahoo.com Menno-Jan Kraak, University of Twente, Enschede, the Netherlands, m.j.kraak@utwente.nl Simone Lanucara, IREA CNR, Milan, Italy, lanucara.s@irea.cnr.it Luciene Lazari, Full Professor, Curitiba, Brazil, luciene@ufpr.br Songnian Li, Ryerson University, Toronto, Canada, snli@ryerson.ca Peter Loewe, German Institute for Economic Research - DIW Berlin, Berlin, Germany, ploewe@diw.de Pierre-Philippe Mathieu, ESA, Frascati, Italy, Pierre.Philippe.Mathieu@esa.int Marco Minghini, Politecnico di Milano, Milan, Italy, marco.minghini@polimi.it Helena Mitasova, North Carolina State University, , USA, hmitaso@ncsu.edu Peter Mooney, University or Research Center: Maynooth University, Maynooth, Ireland, peter.mooney@mu.ie Rafael Moreno-Sanchez, University of Colorado Denver, Denver, USA, Rafael.Moreno@ucdenver.edu Elifuraha Mtalo, University of Bagamoyo, Dar es Salaam, Tanzania, eg_mtalo@yahoo.com Hristo Nikolov, Space research and technology institut, Sofia, Bulgaria, hristo@stil.bas.bg Daniele Oxoli, Politecnico di Milano, Milan, Italy, daniele.oxoli@polimi.it Antoni Perez-Navarro, Universitat Oberta de Catalunya, Barcelona, Spain, aperezn@uoc.edu Andrea Podor, Óbuda University, Székesfehérvár, Hungary, podor.andrea@amk.uni-obuda.hu Kalum Priyanath, Scientific Research Development Institute of Technology Australia, Loganlea, Australia, kalumu@srdita.com.au Venkatesh Raghavan, Osaka City University, Osaka, Japan, raghavan@media.osaka-cu.ac.jp Sven Schade, EC-JRC, Ispra, Italy, S.SCHADE@ec.europa.eu Charlie Schweik, University of Massachusetts, Amherst, USA, cschweik@pubpol.umass.edu Richard Sliuzas, ITC Netherlands, Enschede, Netherlands, sliuzas@itc.nl Naa Tagoe, University of Mines and Technology, Tarkwa, Ghana, ndtmantey@umat.edu.gh Tuong-Thuy Vu, Hoa Sen University, HoChiMinh, Vietnam, thuy.vutuong@hoasen.edu.vn Mayra Zurbarán, Universidad del Norte, Barranquilla, Colombia, mzurbaran@uninorte.edu.co Darshana Rawal, Center for Advance Geomatics, CEPT University, Ahmedabad, India, rawalnet@cept.ac.in Ana-Maria Raimond, IGN, LASTIG Laboratory, Saint-Mandé, France, ana-maria.raimond@ign.fr Paolo Corti, Center for Geographic Analysis, Harvard University, Cambridge, MA, USA, pcorti@fas.harvard.edu Berk Anbaroglu, Hacettepe University, Ankara, Turkey, banbar@gmail.com Sultan Kocaman, Hacettepe University, Ankara, Turkey, sukocaman@gmail.com Anthonia Ijeoma Onyeahialam, Department of Geography and Earth Sciences - Aberystwyth University, Aberystwyth, UK, aio@aber.ac.uk

Chris Stewart, European Space Agency, Frascati, Italy, chris.stewart@esa.int

\section{FOSS4G 2018 Academic Track Chairs}

Maria Antonia Brovelli, Politecnico di Milano, Milan, Italy, maria.brovelli@polimi.it Ally Hassan Namangaya, Ardhi University, Dar es Salaam, Tanzania, namangaya@yahoo.com 